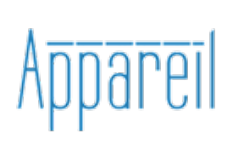

Appareil

$19 \mid 2017$

Friedrich A. Kittler : Esthétique et théorie des médias

\title{
Archéologies sauvages : Freud et Foucault au péril de Kittler
}

\section{Knut Ebeling}

Traducteur : Slaven Waelti

\section{(2) OpenEdition \\ Journals}

\section{Édition électronique}

URL : http://journals.openedition.org/appareil/2537

DOI : 10.4000/appareil.2537

ISSN : 2101-0714

Éditeur

MSH Paris Nord

Référence électronique

Knut Ebeling, "Archéologies sauvages : Freud et Foucault au péril de Kittler », Appareil [En ligne], 19| 2017, mis en ligne le 02 janvier 2018, consulté le 30 juillet 2020. URL : http://journals.openedition.org/ appareil/2537; DOI : https://doi.org/10.4000/appareil.2537

Ce document a été généré automatiquement le 30 juillet 2020.

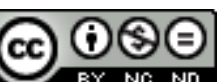

Appareil est mis à disposition selon les termes de la Licence Creative Commons Attribution - Pas d'Utilisation Commerciale - Pas de Modification 4.0 International. 


\title{
Archéologies sauvages : Freud et Foucault au péril de Kittler
}

\author{
Knut Ebeling \\ Traduction : Slaven Waelti
}

\section{NOTE DE L'ÉDITEUR}

Ce texte reprend, sous forme abrégée et remaniée, un chapitre de Wilde Archäologien 1.

Theorien der materiellen Kultur von Kant bis Kittler (Berlin, Kadmos, 2012).

\section{La radicalisation de l'avant-garde}

1 L'archéologie des médias propose une réflexion contemporaine sur les médias qui est désormais devenue si commune que des expositions d'art numérique se parent de ce titre ${ }^{1}$. Mais cette reconnaissance généralisée est trompeuse : l'archéologie des médias représente la variante la plus actuelle et dans le même temps la plus controversée d'une approche archéologique ${ }^{2}$. Si la position de Foucault avait déclenché une guerre culturelle dans les années 1960, deux décennies plus tard c'est Kittler qui polarisait à son tour la communauté scientifique. Son archéologie des médias constituait certes une réaction farouche aux sciences herméneutiques de la République Fédérale d'Allemagne à l'issue de la Seconde Guerre mondiale. La rengaine programmatique dont le Kittler de 1980 faisait son étendard était d'Exorciser l'homme des sciences humaines ${ }^{3}$ - exorcisme qui prétendait liquider les sciences humaines et faire apparaître comme « short » leur « long story ». Au lieu de prendre l'interminable chemin des humanités, Kittler empruntait le raccourci (souvent trop court) de l'évidence matérialiste. Dans l'ombre des sciences humaines, il ne découvrait pas des digressions sans fin, mais la discontinuité des médias techniques. Le monde rutilant des sciences, des techniques et des médias avait le plus souvent été ignoré par les sciences humaines. Se tourner vers lui était le geste central du technicien de la culture et de tous ceux qui le suivirent. De fait, leur geste 
consistait dans un retournement tout simple, destiné à rendre visible ce monde caché et en effet, ce geste tout simple est depuis devenu si prégnant qu'il a intégré le jargon de l'archéologie des médias : il appartient au "Kittler-Sound ${ }^{4}$ ». Le petit "pas de côté5 » peut tout transformer et faire apparaitre une nouvelle image du savoir.

2 Au regard de cette orientation décisive, une tendance qui s'est formée dans la récente tradition de la supposée « école kittlérienne » peut paraître étonnante : la tendance à rattacher l'histoire des médias à cette histoire de la théorie, voire de la philosophie dont elle s'était précisément détournée de manière si décidée. Ainsi, à partir des positions de Kittler, on a récemment entrepris des efforts comparables à ceux qui, en leur temps, avaient jeté le trouble parmi les disciples de Foucault. Le signe le plus évident de tels efforts (à mon sens parfaitement légitimes) est le balisage d'une ligne kantienne, pour ne pas dire transcendantale, de l'histoire des médias. Cette dernière se trouve impliquée dans une "guerre de succession larvée pour le trône vacant de l'a priori $^{6} »$. Et depuis peu, on va jusqu'à vouloir asseoir les médias sur ce trône vacant. L'histoire des médias n'analyse-t-elle pas de fait les «conditions postkantiennes et postfoucaldiennes de la possibilité de l'expérience ${ }^{7}$ "? On ne saurait formuler une exigence de manière plus claire. L'expression d' « a priori technique ${ }^{8}$ " démontre déjà suffisamment que les recherches actuelles prennent bien la direction que ces premières tentatives théorico-historiques dessinaient. Pour autant, l'archéologie des médias ne peut être rangée sans hésitation dans une tradition que l'on pourrait qualifier d'« avant-gardes archéologiques », ou d'« archéologies sauvages" » dont l'arc irait de Kant jusqu'à Kittler - ce qui, il faut en convenir, est encore difficile à articuler. Ce rapprochement osé démontre à lui seul que le diagnostic historico-théorique est tout sauf un désamorçage. La véhémence réactionnaire de l'archéologie des médias ellemême ne peut être évaluée correctement qu'en regard des "avant-gardes archéologiques" précédentes. En cela, ce n'est pas un hasard si Kittler trouve précisément sa place dans la tradition de ces "archéologies sauvages » auxquelles il s'est pratiquement exclusivement référé. Ce n'est pas un hasard s'il s'appuie sur Foucault et Freud, deux points de départ qui sont aussi archéologiques l'un que l'autre. Ce n'est pas un hasard non plus si à ces points de départ s'ajoute, avec l'archéologie des médias, la version ultime de ces "avant-gardes archéologiques». Une séquence théorique claire se dessine qui va de Freud à Kittler en passant par Foucault. Ce que l'archéologie des médias combine, ce sont les points de départ de la psychanalyse et de l'analyse du discours d'une part, de la théorie des médias et de l'épistémologie de l'autre. Avec cette combinaison, se surimpose à l'histoire du discours archéologique une variante contemporaine - et l'impossible est rendu possible: l'énigmatique archéologie du discours de Foucault s'en trouve encore affûtée. L'archéologie des médias se donne pour tâche « de partir de Foucault pour pousser analytiquement audelà de Foucault - et pour savoir archéologiquement les médias ${ }^{10} »$. En d'autres termes : l'avant-garde foucaldienne devient encore plus radicale. L'archéologie du savoir est aiguisée en une archéologie médiatique du savoir ou archéologie des médias. Avec Kittler, l'épilogue foucaldien de la disparition de l'homme se transforme en une nécrologie du point de vue des machines qui l'enterrent. En un mot: le ralliement de Kittler à Foucault est l'un des rares prolongements de la French Theory qui ne la banalise ni ne la relativise - mais au contraire la radicalise ${ }^{11}$. 


\section{Le refoulement des médias}

3 L'avant-garde média-archéologique part du diagnostic selon lequel les techniques, les médias et les histoires des sciences ont été victimes d'une ignorance coupable de la part des sciences humaines traditionnelles. Les médias et les techniques furent refoulés par l'esprit hégélien, tout comme le fut également la préhistoire, selon le mot de Bataille. Partant de cette hypothèse, l'archéologie des médias s'est tout d'abord fondée sur une théorie du refoulement transformée en épistémologie: Kittler part de l'idée selon laquelle les sciences humaines ont historiquement et systématiquement refoulé le médium et la technique en dehors de leur domaine. Certes, des chercheurs en sciences humaines de plus en plus hébétés s'intéressent de temps en temps au contenu des médias ; mais précisément parce qu'ils portent leur regard sur leur contenu, ils doivent nécessairement en perdre de vue les conditions matérielles - en dernier recours, les chercheurs en sciences humaines formés à l'école de l'herméneutique lisent des relations de sens, et non des colonnes de lignes, ils voient des images de films et non des tubes cathodiques. Les contenus médiatiques se glissent ainsi en un sens devant les appareils. Les médias occultent en même temps qu'ils montrent. La technique des médias est donc refoulée non en dépit mais en raison du fait que l'on prend de plus en plus en compte leur contenu. Tel est le dilemme de toute théorie des médias : plus on porte son attention sur les médias, plus leur hardware (le slogan central de Kittler) disparaît irrémédiablement du champ du regard. Les humanités s'établissent dans l'exclusion de tout ce qui relève de la technique, qui est pourtant partie prenante de leur savoir. Le retour de ce refoulé s'est dès lors fait plus virulent que les administrateurs du savoir ne l'auraient souhaité.

Le scénario habituel se noue : le constat d'un refoulement des techniques et des médias fournit à leur archéologie l'argument central d'une campagne vengeresse: c'est précisément parce que les sciences humaines se sont rendues coupables du refoulement complet des techniques et des médias dans le sous-sol de leur savoir, qu'une archéologie put se consacrer à rechercher ce refoulé. Au plus tard avec ces fouilles qui rattachèrent le fonds enfoui des médias à l'archéologie freudienne de la psyché, la théorie du secret de l'inconscient débouche sur une théorie généralisée du complot. En tant que telle, l'archéologie des médias se distingue radicalement de toute compréhension éclairée des médias : on n'avait du reste pas attendu Strukturwandel der öffentlichkeit de Habermas - dont le titre français Archéologie de la publicité est plus que curieux $^{12}$ - pour comprendre les médias comme une institution émancipatrice. En tant qu'institution sociale publique, ils confèrent à la société la transparence dont toute démocratie a besoin. Mais dans le même temps, la conception d'un élément secret produit par les médias fragilise les théories idéologiques et critiques des médias qui partent du principe que ces derniers doivent informer dans un esprit de vérité, mais seraient instrumentalisés au cours du développement public de leur pouvoir.

5 Au contraire de ces approches émancipatrices, l'archéologie des médias fait migrer l'idée de manipulation dans le concept même des médias. Elle n'étudie pas la presse, la radio, la télévision sous l'angle de la sociologie ou de la communication de masse l'archéologie des médias pense les machines et technologies impensées et inaperçues, cachées par les formes publiées. En un mot: l'archéologie des médias n'adopte pas le point de vue de la sociologie ou de la philosophie, de la communication de masse ou de la psychologie sur les médias ; elle observe au contraire, du point de vue des médias, les 
productions culturelles (et non-culturelles) de tous genres. En cela les sociologies, les philosophies et les psychanalyses se trouvent submergées par la matérialité des médias qu'elles avaient ignorée, oubliée ou refoulée. La figure archéologique du fonds enfoui ou du refoulé refait ainsi surface par le biais des médias : parce que leur archéologie portait en premier lieu son attention sur le savoir, elle put, au rebours de l'histoire des médias, découvrir que ce savoir enfouit résolument les conditions matérielles et médiales de sa production. Tandis que les médias masquent habituellement dans leurs fonctions leurs opérations techniques, l'archéologie des médias tente de rendre à nouveau lisible ces "opérateurs de l'écriture ${ }^{13}$ » dissimulés. L'archéologie des médias s'est donné pour tâche de rendre à nouveau visibles les opérations matérielles et mathématiques qui avaient été recouvertes par des sciences humaines centrées sur le contenu et l'interprétation. Aux yeux des représentants de ces disciplines qui ignorent systématiquement la technique, les médias sont enfouis et nécessitent des fouilles archéologiques. En cela, les premières archéologies des médias impliquent toujours aussi l'archéologie de ces sciences humaines et littéraires qui avaient refoulé ces médias en premier lieu - pour cette raison, la mise au jour du refoulé coïncide également avec la tentative de «transposer et sauver techniquement la germanistique pour le $\mathrm{xx}^{\mathrm{e}}$ siècle $^{14} »$.

6 Qu'est-ce qui constitue le fonds enfoui et le refoulé des médias? Quel est l'objet de leur archéologie ? D'une part, l'archéologie des médias pense ce que l'histoire de l'esprit enterre le plus farouchement: son imprégnation discrète par la matérialité des techniques et des médias. Et si l'on veut faire passer pour sublimés et spiritualisés les textes de la tradition, leurs magies immatérielles demeurent l'indice de leur mode d'existence technique. L'archéologie des médias agit ainsi selon la règle d'or qui veut que plus un produit culturel se donne pour sublimé, plus il est certain que des techniques et des médias - qu'il occulte et refoule ${ }^{15}$ - ont contribué à le former. Pour autant, ce ne sont pas les médias qui (du moins dans les approches idéologiques et critiques) occultent leur origine en la dissimulant derrière du contenu. L'archéologie des médias part du principe que tous les types de produits culturels - littérature, art ou science - refoulent les appareils médiatiques à l'aide desquels ils sont mis au monde.

7 Le regard sur les appareils médiatiques et sur les matérialités suffit-il ? Comment les choses fonctionnent-elles par exemple pour l'ordinateur? Un premier regard sur l'objet par excellence de l'archéologie des médias montre que les médias numériques ne se laissent pas réduire à la matérialité de la technique : on n'explique pas un ordinateur uniquement par ses câbles, boîtiers et processeurs. L'archéologie de l'informatique ne saurait consister à fouiller les décharges de ce monde à la recherche de boîtiers d'ordinateurs. On doit y adjoindre ces mathématiques fondamentales qui seules permettent de faire tourner un ordinateur. La première chose à être enfouie - cela Kittler le comprend plus clairement que Benjamin - c'est la finitude mathématique de leur matérialité. Et ce qui, de cette archéologie de la finitude, doit être mis au jour, c'est "l'événement matériel appelé reset ${ }^{16} »$. L'objet de l'archéologie des médias est de la même manière mathématique et matériel. Les innombrables archéologies de l'ordinateur doivent étudier à la fois l'histoire de la technique et celle des mathématiques ${ }^{17}$.

8 L'archéologie des médias est redevable d'un paradoxe: les médias ne font pas que communiquer, ils dissimulent également. Ils enfouissent en montrant - et tandis qu'ils montrent, envoient, transmettent et stockent, le message stocké et transmis, envoyé et 
montré, masque les médias au fondement de ces fonctions. Dans notre modernité avancée, ce sont les médias qui non seulement montrent, mais également occultent les choses: «le support de l'archive est originairement soustrait au regard de l'observateur ${ }^{18}$ ». La première chose que les médias camouflent, c'est leur status nascendi, leur propre matérialité. À partir de cette disparition, il est possible d'envisager une archéologie des médias qui réimporte dans le savoir ce que les médias enfouissent. Il s'agit donc bien d'une archéologie également au sens de fouilles d'un fonds enfoui, au sens de dés-encastrement des fonctionnalités perdues et de la matérialité des médias. Sur ce point également, le geste subversif de l'archéologie des médias est redevable d'un discours archéologique : c'est uniquement parce qu'à la suite des " avant-gardes archéologiques ", elle a adopté la théorie d'un fonds enfoui, qu'elle est la seule parmi les théories actuelles des médias à pouvoir indiquer de manière complotiste le redoublement de l'ostension et de l'occultation. Peut-être que les archéologies des médias vont jusqu'à produire ce qu'elles dés-encastrent dans le mouvement par lequel elles réalisent leurs fouilles : en dernier lieu, leur capital repose bien sur la capacité à désenchanter le fonds enfoui qu'elles génèrent elles-mêmes sans relâche ${ }^{19}$.

\section{L'inconscient technique}

Il n'y a pas que les patients qui refoulent, des cultures entières s'y emploient. Comme les expressions et les souvenirs des sujets, celles des cultures également dépendent de la dynamique du visible et de l'enfoui. D'une part, ce diagnostic conduit aux théories connues de la mémoire sociale, collective ou culturelle. D'autre part, il fournit un argument à l'archéologie des médias qui se rattache directement à l'archéologie de la psyché : parce que les produits culturels travaillent en refoulant, comme Freud l'avait remarqué chez ses patients, l'archéologie doit traiter les productions culturelles comme des patients - et les coucher sur le divan. Il n'y a pas que la conscience qui puisse être déchiffrée comme des tablettes d'argile. L'analyse des petites tablettes d'argile et des consciences peut servir de modèle à l'analyse du discours et des médias. L'archéologie des médias couvre les opérations archaïques et primitives des médias que voilent leurs contenus. Pour cette raison, Kittler traite de Scènes primitives (1977), de "préhistoires " et de "l'âge de pierre de l'ordinateur »: le clou de l'archéologie de l'ordinateur consiste à étudier le plus avancé des médias en adoptant la plus archaïque de toutes les approches - et du même coup de secouer d'une chiquenaude l'âge de l'ordinateur.

Ce n'est pas sans raison qu'à côté de Foucault, Freud soit le deuxième témoin principal de l'archéologie des médias ${ }^{20}$. Cette approche combine plusieurs traditions: les décentrements de Heidegger et de Foucault comme la pensée des médias de Benjamin et de McLuhan d'une part - et d'autre part une psychanalyse initiée par Freud et prolongée par Lacan ${ }^{21}$. La proximité de Kittler avec Freud saute certes moins aux yeux que celle avec Foucault (à qui Kittler a probablement emprunté la volonté de se distancier de la psychanalyse). Cette proximité demeure cependant évidente. Dès ses premières publications, Kittler traite constamment des rêves et de la parole, des mères et des bouches ${ }^{22}$. Et rapidement, il convoque même l'icône des «archéologies sauvages ", la Gradiva de Freud ${ }^{23}$. En un tour de main, la muse de l'archéologie de l'âme devint la sirène de celle des médias. Ce transfert d'image souligne le statut de l'histoire 
des médias comme une science à venir en même temps que ce qu'elle doit à l'analyse du discours et à la psychanalyse.

11 Nous avons déjà évoqué la théorie d'un refoulement des médias par les sciences humaines. Le refoulé médiatique est élevé au rang d'objet par l'archéologie des médias de la même manière que la psychanalyse avait thématisé le refoulé psychologique. Or plus encore que la théorie du refoulement, ses objectivations trouvent leur origine dans les «avant-gardes archéologiques»: Benjamin avait adapté le refoulé invisible au registre visuel de l'«inconscient optique ${ }^{24}$ » de la photographie. Et la spécificité médiatique de ce refoulé optique a été transformée à son tour par Foucault en un « inconscient positif du savoir ${ }^{25}$ ». À la fin de la série des objectivations de l'inconscient, l'archéologie des médias fit la découverte de l'inconscient technique du savoir. Malgré cette succession, il existe bien une différence fondamentale dans la manière dont Freud et Foucault sont rattachés aux " archéologies sauvages ». Foucault n'est devenu que peu à peu l'objet de l'archéologie des médias; Freud en revanche en fut l'un des premiers objets: l'archéologie de la psyché a été partie prenante du développement de l'archéologie des médias.

12 En prenant un peu de hauteur, on pourrait considérer qu'il s'agit là d'une transformation machinale de sujets méthodiques en objets de recherche: après que Kittler ${ }^{26} \mathrm{a}$ interprété de manière psychanalytique les littératures romantiques sous le signe des célèbres bouches maternelles, la psychanalyse est elle-même devenue l'objet des analyses du discours. Le premier objet de Kittler étudié au titre de «système de prise de notes de $1900^{27}$ » a été L'Interprétation du rêve. Le livre - qui fit date - de Kittler démonte les codes médiatiques de la talking cure avec une précision et une brutalité que l'on ne peut appliquer qu'aux penseurs du passé; et l'on peut en dire autant de la manière dont Kittler s'est saisi de Foucault. Dans le rapport de l'analyse des médias à l'analyse du discours, on diagnostique un déplacement similaire du procédé vers l'objet. Après les Scènes primitives ${ }^{28}$ de l'analyse du discours, même le fondateur de l'archéologie du discours put devenir l'objet d'une archéologie des médias. Là où le sujet était, les objets de l'archéologie des médias doivent advenir. La victime visible de cet exorcisme fut cependant Freud : c'est en s'appuyant sur les mécanismes du travail du rêve qu'une théorie média-technique de l'encodage du savoir était advenue. Et c'est seulement après que la psychanalyse est devenue l'objet d'une analyse du discours, que fut inventé le sujet de l'histoire des médias que le "matérialisme de Freud ${ }^{29}$ " avait anticipé de manière exemplaire. Et c'est seulement alors que l'« appareil psychique » freudien put être décodé comme "implémentant tous les médias de transmission et de stockage disponibles ${ }^{30}$ ». En un mot : de la même manière que l'on peut parler chez Freud d'une théorie du représentable, et chez Foucault d'une théorie du dicible, on peut dans leur double continuité parler d'une théorie du codage chez Kittler.

Certes, des codages étaient déjà apparus dans les analyses du travail du rêve par Freud. Ce n'est toutefois qu'avec les archéologies des médias qu'ils donnèrent lieu à une théorie à part entière, et qu'ils prirent les traits de concepts d'une technique de la communication. L'Archéologie du savoir s'était définie comme un discours sur la transformation des discours ${ }^{31}$. L'archéologie des médias se forme par la transposition de codes en codes. L'objet de l'archéologie des médias est de savoir «quel code est porté par quel médium ${ }^{32} »$. Quel médium permet - code ou guide - quels contenus ? Quelle architecture informatique donne quels ordres? Qu'est-ce qui, à l'intérieur de médias particuliers est dicible dans leur codage, et qu'est-ce qui ne peut plus être dit? 
En portant l'accent sur le savoir, sur ce qui peut être dit et écrit, l'archéologie des médias démontre qu'elle se trouve sur le seuil entre les immatérialités structuralistes et les matérialités poststructuralistes ${ }^{33}$. Certes, parmi les fragments du rêve et entre les couches des épistémès régnaient bien des rapports différentiels, tout comme dans le monde technique des médias. Mais seul Kittler a délivré une théorie du codage en cela qu'il en a quantifié tous les enchaînements en se fondant sur la théorie de l'information de Shannon. En effet, la théorie de l'information de Shannon - « un Foucault doté de capacités mathématiques supérieures ${ }^{34} »$ - peut être comprise comme une formalisation anti-herméneutique de l'analyse du discours. Shannon a déduit la théorie du codage non pas à partir de la langue mais de la technique. À l'instar de Vilém Flusser ${ }^{35}$, il n'a pas ignoré le gouffre profond qui sépare signification et calcul. Shannon ne travaille pas avec la sémantique, mais avec les mathématiques. Ce faisant, il libère la théorie du codage de ses derniers restes métaphoriques. La différence entre texte et technique est fondamentale quand il s'agit «de décrire des codes autres, et qui ont infiltré depuis longtemps le code alphabétique ${ }^{36} \%$. Ce qui ressort du projet de description ce sont «les écritures techniques, et non pas alphabétiques, ce sont les écritures numériques ou algébriques ${ }^{37}$ ».

Kittler, cependant, doit aussi son approche aux archéologues avant-gardistes et pas seulement à l'avant-garde de la science des médias. Son rattachement à la psychanalyse et à l'analyse du discours se construit en trois étapes : de Freud à sa propre archéologie en passant par Foucault (tandis que Benjamin est largement passé sous silence). Les analyses psychanalytiques du travail du rêve avaient permis de mettre au jour des procédés et des techniques à partir desquels certains des bricolages du rêve devenaient représentables, et d'autres pas. L'analyse du discours avait permis de développer les circuits discursifs qui rendaient certains savoirs dicibles, et d'autres pas. Faisant suite à ces analyses, l'archéologie des médias se tourne vers les conditions de ce qui peut être su et de ce qui ne le peut pas sur la base de médias spécifiques ${ }^{38}$. Tout comme il y a chez Foucault des énoncés fondamentaux qui définissent l'archive d'une époque, il y a chez Kittler des techniques fondamentales qu'il s'agit de mettre au jour. En vertu du principe foucaldien "du resserrement ${ }^{39}$ ", il ne s'agit jamais que d'un nombre limité d'opérations techniques fondamentales qui s'effectuent « dans notre dos ${ }^{40} »$.

\section{L'archéologie médiatique du savoir}

15 La pertinence de l'archéologie des médias ne se révèle cependant que lorsqu'on ne la lit pas dans la continuité d'une enquête sur les discours, mais comme une polémique contre des sciences humaines bien ronflantes. L'archéologie des médias se met en scène comme une irruption brutale des techniques culturelles et des matérialités médiatiques dans les bibliothèques des sciences humaines. Dans l'archéologie des médias, les techniques définissent les procédés par lesquels on peut saisir le codage d'une production culturelle dans son ensemble. Et cela veut dire que le mouvement médiaarchéologique évoqué plus haut, qui consistait à faire de sujets des objets du savoir, a lui-même été retourné en son contraire : les médias ne sont plus les objets mais les sujets de la recherche. Ils changent de catégorie: d'objets observés, ils deviennent méthode d'analyse du savoir culturel. Dans l'archéologie des médias, les médias se déplacent du côté des procédés du savoir. Son objet n'est plus seulement les médias, ces 
derniers indiquent au contraire le procédé selon lequel il convient d'analyser les multiples formations du savoir.

16 Ce changement de statut des médias a la même signification que la différence entre histoire des médias et archéologie des médias : une histoire des médias raconte la succession chronologique des médias, prenant place sur une ligne temporelle en se fondant sur des documents (c'est-à-dire des sources écrites) matériels. Une archéologie des médias est plus fondamentale : se fondant sur la matérialité et sur la technique des médias, elle essaye de définir un nouveau classement du monde écrit, des documents et du savoir. L'archéologie et l'histoire des médias se distinguent sur deux points : par rapport à leurs matérialités et par rapport à leurs objets. Dans l'histoire des médias, ces derniers servent d'objets visibles dont on écrit l'histoire au même titre que celle des transports ou du téléphone. Dans l'archéologie des médias au contraire, les médias glissent de la position d'objet à la position de procédés de la recherche. Les médias prennent la place du sujet pour classer à neuf les objets de l'histoire et du savoir. Au lieu d'étudier la préhistoire du téléphone, l'archéologie des télécommunications étudie ses effets épistémiques ${ }^{41}$. L'archéologie analyse les médias comme producteurs du savoir ; elle pense le fonds matériel enfoui du processus médiatique du savoir.

Ce travail épistémique sur le fonds enfoui distingue l'archéologie des médias, premièrement, de toute élaboration faisant intervenir une infra et une superstructure (selon laquelle les médias constitueraient l'infrastructure qui détermine la superstructure sociale ${ }^{42}$ ). Deuxièmement, le travail sur le fonds enfoui distingue l'archéologie des médias d'une histoire des médias qui s'intéresse à leurs développements historiques. Pour le dire de manière polémique : l'histoire des médias traite des noms et des données de l'invention des médias - et pas de leur fonction effective et matérielle. Elle travaille sur une histoire des médias toujours déjà visible et pas sur les conditions médiatiques invisibles qui structurent le savoir. En un mot, l'archéologie des médias ne s'intéresse pas seulement aux médias, mais également aux divers objets culturels dans une perspective médiatique. Elle ne pose pas la question des médias comme produits d'une culture, mais au contraire la question de la production. Parce que «tout ce qui de [l'art et de la philosophie] est culturellement transmis, est également commutable ${ }^{43}$ ", les analyses que l'archéologie des médias fait d'un savoir dans une perspective technique et médiatique sont quelque chose de totalement différent d'une théorie ou d'une histoire des médias. Tandis que l'histoire des médias fixe les médias dont elle veut écrire l'histoire, l'archéologie des médias s'intéresse moins aux médias qu'au savoir qu'ils produisent. En dernier lieu, «il se passe un nombre effroyable de choses entre le silicium et son output psychique ${ }^{44} »$.

Le silicium et ses effets psychiques - on pourrait déchiffrer ce couplage comme une nouvelle image ou, à tout le moins, comme une nouvelle configuration du savoir: en premier vient le médium et ensuite ses effets (par exemple psychiques). Les médias sont ici considérés comme les moteurs ou les machines d'un savoir qui travaille généralement dans le fonds enfoui ; avec cette machine les « outputs psychiques » qu'ils produisent deviennent étudiables. L'archéologie des médias regarde un objet nommé "savoir » du point de vue des restes médiatiques. Son " étude en profondeur " (Freud) du savoir culturel s'intéresse à ses formations médiatiques et techniques. Et parce qu'il "se passe un nombre effroyable de choses entre le silicium et son output psychique », le rapport entre le médium et le savoir n'est pas le rapport causal à l'échelle 1:1 entre une infrastructure et une superstructure ${ }^{45}$. 
19 d'une logique contingente. Cette contingence peut être rapprochée de la logique des émergences non-causales que Benjamin a mise en lumière dans le phénomène de la mode. Comme ces dernières, les médias forment des conditions particulières du savoir qui rendent certaines choses dicibles et d'autres pas. Benjamin avait essayé de saisir par-là les lois de l'apparition et de la disparition de choses culturelles - raison pour laquelle la différence entre Benjamin et Kittler peut être rapportée exactement à la différence entre une archéologie qui opérerait encore comme une science historique et humaine, et une archéologie des médias qui opérerait techniquement. L'héritage philosophique de l'archéologie de la modernité a lui-même été assujetti à l'archéologie médiatique de la contingence a-historique et discontinue des standards techniques. Dès lors l'histoire n'est plus fondée de manière causale, mais elle est calculée mathématiquement. Ces calculs ne sont pas la conséquence de causalités historiques, mais de modes de calcul qui auraient aussi bien pu être différents ${ }^{46}$.

Un second reproche qui est parfois formulé à l'encontre de l'archéologie des médias est celui du réductionnisme médiatique. La différenciation entre l'histoire des médias et l'archéologie des médias a ici toute son importance, car le reproche toucherait bien une histoire des médias limitée aux objets médiatiques ${ }^{47}$. L'archéologie des médias en revanche y échappe ne serait-ce qu'en raison de son héritage épistémologique : elle ne se limite pas à étudier les médias en tant que tels, elle étudie divers objets culturels dans la perspective des médias. Pour l'archéologie des médias, il s'agit d'en analyser le rôle dans l'enfouissement du savoir - et non pas de l'analyse de médias donnés. Pour cette raison, on peut également parler d'une archéologie médiatique du savoir ${ }^{48}$. Dans cette archéologie médiatique, les médias ont pris la place de l'«inconscient positif du savoir ${ }^{49}$ " sur lequel l'archéologie des sciences humaines avait déjà fait son rapport. Avec ce déplacement, l'inconscient est premièrement plus radicalement positivé que chez Foucault; deuxièmement la positivité indépassable des standards techniques prend la place de ces constances qui, dans les facultés de philosophie, firent longtemps l'objet d'introductions méthodiques. Les sujets philosophiques sont remplacés par les standards techniques; au plus tard dans les Aufschreibesysteme ${ }^{50}$ les équations mathématiques remplacent les introductions philosophiques ${ }^{51}$. Les standards discontinus et immuables des médias techniques remplacent les sujets maîtres de l'histoire - en conséquence de quoi les introductions philosophiques pourraient disparaître des œuvres standard de l'histoire des médias, comme disparaissent les rois dans les guerres de succession.

21 Tel fut le chemin qui mena d'une archéologie du savoir à une archéologie des médias: Foucault avait montré que le sujet ou l'homme n'est jamais directement atteignable, mais toujours via le détour du savoir et des énoncés. À la suite de Foucault, Kittler démontre que ces énoncés ne sont en rien indépendants ou autonomes. Non seulement les actes de langage, les contextes et les discours participent à la constitution du savoir ; mais les médias également. Pour cette raison, on peut donc passer directement d'une analyse du savoir à une analyse des conditions médiatiques de ce savoir. La question des conditions médiatiques des formations du savoir renvoie bien en dernier lieu à l'héritage épistémologique de l'archéologie des médias. C'est seulement après l'établissement de ce monde intermédiaire de constructions ou "formations " - en référence à La Formation de l'esprit scientifique de 1938 par Bachelard -, qu'il devient possible de pratiquer l'analyse des médias au niveau de l'épistémologie et de l'analyse 
du discours. La question vise par conséquent toujours la partie médiatique ou technique, ou les composantes du savoir : quel savoir a-t-il été possible dans quelles constellations médiatiques particulières? Qu'a-t-on pu écrire dans les conditions de quels systèmes techniques de prise de notes? Quel savoir est-il devenu possible à partir de quel moment donné? avec quel médium particulier? dans quelles conditions techniques? et quel savoir ne le fut pas?

\section{La tache aveugle de Foucault}

L'archéologie médiatique du savoir prolonge l'archéologie du savoir à un nouveau niveau. Le rapport entre ces deux projets est-il comparable à celui que Foucault avait décelé entre La Critique de la raison pure et l'Anthropologie d'un point de vue pragmatique? Kittler répète-t-il le projet de Foucault au niveau empirique (et cela signifie ici évidemment, au niveau médiatique) ? Le transcendantal est-il transposé par Kittler du niveau historique de Foucault au niveau médiatique? En tout état de cause, il convient de tenir compte de l'héritage transcendantal que l'archéologie médiatique antikantienne du savoir a fait sien; au plus tard avec la mise en œuvre de l'a priori technique on en revient à ce point surprenant. Il n'est en aucun cas possible de réduire l'entreprise de Kittler à une histoire des objets médiatiques, comme cela se voit parfois. Il s'agit d'un prolongement de Foucault et en cela d'une archéologie - de telle sorte que le geste de Kittler envers Foucault « hésite entre correction, succession et rédemption ${ }^{52}$ $»$.

23 Ici apparaît donc une différence à l'intérieur d'une continuité, telle qu'elle était déjà apparue dans le rapport de Foucault à Kant. Au premier plan se trouve la continuité : le geste nietzschéen fondamental de scepticisme historique et de subversion, tel qu'il s'est depuis 1980 manifesté dans Exorciser l'homme des sciences humaines est parvenu à Kittler via Foucault. Un nombre conséquent de remarques, dédicaces, préfaces et postfaces attirent l'attention sur la présence des lectures de Foucault ${ }^{53}$. Kittler a repris de Foucault une pensée se mouvant entre des éléments discursifs et non discursifs, tout comme des conceptions centrales telles que l'«archive» ou le «savoir». Le concept foucaldien d'énoncé est littéralement réévalué comme mode d'enregistrement technique. Sa notion de praxis est déclinée à travers les pratiques médiatiques particulières : de la même manière que Foucault ne demandait pas ce que les textes signifient, mais ce qu'ils font de leurs lecteurs, Kittler enquête sur les conditions de réalité des médias.

Mais des différences apparaissent également à l'intérieur de la continuité. Elles apparaissent déjà dans le déplacement de textes et discours comme agents du savoir, vers les médias : l'archéologie des médias n'est pas que la « suite » de l'archéologie du discours, mais bien sa « correction » et sa « rédemption ». En raison de ces différences à l'intérieur de la continuité, Kittler put prendre la pose d'un «nain sur les épaules d'un géant ${ }^{54}$ ", qui voit de fait plus loin que ce dernier. Or, au contraire de ce que cette image suggère, il n'y eut jamais de rencontre entre le nain et le géant. Certes, ils furent tous deux au même endroit au même moment en 1980, lors de la dernière du Ring par Patrice Chéreau à Bayreuth - Foucault venait de donner dans Le Monde sa célèbre interview anonyme comme "philosophe masqué55". La rencontre fut une nonrencontre. Tandis que la mise en scène légendaire de Chéreau inspirait à Foucault un texte enthousiaste sur Wagner, "L'Imagination du $x^{e} x^{e}$ siècle $^{56}$ ", Kittler se laissait 
quant à lui aller à écrire un paragraphe entier sur Foucault et Kittler ${ }^{57}$ - dans lequel il se nomme ouvertement dans un seul souffle avec le géant. Dans cette non-rencontre, Kittler maintint envers Foucault une distance comparable à celle que Freud avait maintenue envers Dörpfeld, l'assistant de Schliemann ${ }^{58}$; une distance qui n'est du reste que l'envers de lectures d'autant plus avides ${ }^{59}$. Plus l'aveu de proximité est profond, plus la distance est nécessaire. Dans le rapport à Foucault, la distance dût être, pour Kittler, aussi inévitable qu'elle ne l'avait été pour Foucault dans son rapport à Freud. C'est là la seule manière d'expliquer que Foucault, qui était traité alors comme l'exécuteur le plus radical de l'histoire, en devint pour Kittler l'agent compilateur. Au geste de succession s'ajoute un geste de correction.

Les analyses de Kittler ont lieu après et d'après Foucault : Kittler suit Foucault tant sur le plan de l'histoire que sur celui de la méthode. Tandis que nous traiterons de la succession historique de Foucault dans la prochaine section, nous voulons aborder ici le prolongement méthodologique de l'archéologie du discours. En effet, un nombre important de remarques signale l'héritage méthodique de l'analyse du discours dans l'archéologie des médias ${ }^{60}$. Le programme de Kittler est une « analyse du discours dont les éléments ne sont visiblement plus seulement les mots mais encore le code ${ }^{61}$ ", une méthode qui opère "dans un champ strictement technique selon des procédés semblables à ceux que l'analyse du discours de Foucault a proposés pour les discours et les textes». Sa «réorientation intérieure» consista à transposer "l'approche foucaldienne des textes ou discours aux médias ${ }^{62} »$. Et si l'on définit l'archéologie des médias comme une boucle de l'analyse du discours au niveau technique - et donc à un niveau extérieur à la pensée - cela induit l'idée que Kittler, le premier, serait parvenu à cette pensée du dehors que Foucault avait annoncée. Ici, « la langue parlée, qui fut jadis dans les oreilles des philosophes l'auto-affectation de la conscience, perd toute intériorité et devient aussi mesurable que toutes les qualités de transmission de système radiophoniques ou télévisuels ${ }^{63} »$.

La continuation de Foucault par Kittler poursuit donc des motifs à la fois psychologiques, méthodiques et stratégiques: l'élargissement média-technique de l'analyse du discours ne peut être mené que par une approche qui a tout autant oublié ou refoulé cet élément que les sciences humaines auxquelles s'en prend Kittler. Un disciple autoproclamé prend donc congé de Foucault avec le même reproche qu'il avait adressé aux sciences humaines - raison pour laquelle «les merveilleuses quantifications des cultures européennes dans dix ou vingt ans [...] tomberont sous le coup des contes des sciences humaines contre lesquelles elles avaient été écrites ${ }^{64} 》$. L'archéologie des médias surdétermine l'archéologie du savoir en se servant du même argument dont s'était déjà servi Foucault pour s'en prendre aux sciences humaines. Kittler retourne clairement l'argument anti-sciences humaines de Foucault contre son auteur.

On peut trouver cette stratégie de surdétermination avec ses propres armes conséquente, ou peu imaginative, voire ingrate. Ce geste vise à permettre à Foucault de parvenir à une vision claire de sa propre théorie : Kittler annonça en effet avoir trouvé la tache aveugle du projet archéologique de Foucault ${ }^{65}$. La tache aveugle de Benjamin, c'étaient les développements théoriques contemporains dans les domaines de l'épistémologie et de l'historiographie. La tache aveugle de Foucault consistait dans le développement des médias. Comme toute tache aveugle, les médias étaient compris également chez Foucault dans une dialectique entre occultation et apparition. La tache 
aveugle ne voile pas seulement quelque chose, la dissimulation a également pour effet de mettre une chose en évidence. D'une part la tache aveugle empêchait d'approcher les médias de manière appropriée; d'autre part elle permit la mise en évidence des discours. C'est seulement dans cette constellation spécifique que Kittler put mettre en scène son programme comme une conséquence et un prolongement strict d'une logique qui était demeurée invisible dans l'analyse du discours: une poursuite de l'archéologie des médias par d'autres moyens. En d'autres termes: l'impression d'une relève impérative est redevable au "truc " de Kittler de présenter "sa réorientation intérieure [...] des textes ou des discours vers les médias [...] comme l'accomplissement d'un programme théorique ${ }^{66}$ ", et comme l'exécution exotérique d'un programme ésotérique préparé par Foucault. Lorsque Kittler défend un "structuralisme des matérialités ${ }^{67}$ ", il met en scène l'archéologie des médias comme accomplissement d'un mouvement de matérialisation du savoir qui avait commencé sur la rive française du Rhin, et qui s'accomplit sur sa rive allemande ${ }^{68}$. La relève de l'analyse du discours n'est donc redevable d'aucune critique externe, mais de son propre programme originaire. C'est en outre ceci qui donna l'impression particulière que la relève demeurait en dernier lieu fidèle à ce programme.

La continuité entre une archéologie du savoir et une archéologie des médias apparaît clairement dans la combinaison de deux traits distinctifs: dans le caractère archéologique et épistémique de l'archéologie des médias. Premièrement, le déplacement des médias comme objets de l'analyse vers une archéologie médiatique du savoir est devenu en premier lieu possible sur la base de l'analyse du discours dont l'objet est également le savoir. Deuxièmement, l'orientation matérielle de l'analyse des médias est redevable de l'approche archéologique qui est, certes, contenue dans les archéologies foucaldiennes, mais qui de l'avis de Kittler n'avait pas encore été réalisée. En un mot : Kittler ne se revendique pas de "la liberté de la théorie française ${ }^{69}$ » d'une manière uniquement abstraite. Il la prolonge d'une manière remarquablement explicite. Il donne l'impression de ne pas être, lui, un Foucault allemand, mais que Foucault aurait au contraire été un « Kittler français ${ }^{70}$ ». D’un nain sur les épaules d'un géant est né un géant qui se tient sur les épaules d'un nain.

Le Foucault surpuissant est réduit à la taille d'un nain dès lors qu'on l'a questionné sur sa théorie des médias. Pour autant, il est problématique de parler d'une "conception des médias ${ }^{71} »$ chez Foucault, telle qu'elle aurait fourni les linéaments d'une théorie des médias qu'il ne s'est jamais proposé de produire. Reprocher à Foucault de n'avoir pas développé de théorie des médias repose ainsi sur une double ignorance : premièrement, celle-ci n'a jamais été envisagée et, deuxièmement, Foucault a bien étudié la littérature à l'intérieur des conditions-cadres d'un médium. De plus, il a bien rencontré au cours de sa carrière les médias au sens de leur archéologie. Des témoins oculaires racontent qu'il avait, en 1951, en sa possession dans son bureau de répétiteur à l'École normale supérieure des tourne-disques 78 tours poussiéreux ${ }^{72}$. Mais l'activité des médias en dehors de leur usage ne fut intégrée dans une conception épistémologique du savoir qu'après Foucault. La nécrophilie des dead media a commencé seulement à partir de l'archéologie de Foucault. C'est seulement à ce moment que d'anciens chercheurs en sciences humaines mirent à exécution la menace de ne plus se limiter à étudier les littératures. 


\section{La défection de l'histoire}

elle est rattachée à un argument historique. Il a déjà été question du fait que l'archéologie des médias tirait sa légitimité d'une dé-légitimation historique de l'analyse $\mathrm{du}$ discours. Cette mise entre parenthèses historique d'un détournement méthodique confère sa pertinence au prolongement culturel et technique de Foucault. J'ai déminé précédemment la critique faite à Foucault en soulignant qu'on ne peut lui reprocher l'absence d'une théorie des médias qui ne fut jamais son but. Pour autant, il nous reste à poser la question critique de savoir si Foucault n'aurait pas dû en arriver aux médias, sachant que son archéologie avait inclus toutes les "conditions-cadres" qui influent depuis le fonds enfoui sur le savoir. Certes, on ne peut peut-être pas reprocher à l'archéologie du savoir d'être devenue la victime de sa propre épistémè. Mais dans la perspective de l'archéologie des médias, les choses semblent bien ainsi : elle est devenue la victime de ces standards techniques qu'elle avait refusé de voir.

'est seulement le couplage de l'argument méthodique à une évolution historique qui confère son autorité à l'archéologie des médias : celle-ci tire sa légitimité - et selon Kittler et Turk cela vaudrait également pour l'analyse du discours ${ }^{73}$ - d'une dynamique historique. Ses analyses en appellent à une césure historique fondée non pas sur les données, mais sur les techniques. L'argument de Kittler est on ne peut plus simple: tandis que l'histoire et ses discours sont indissociablement liés à l'histoire de l'écriture, leur prééminence se clôt également avec la fin de cette dernière - c'est-à-dire autour de $1850^{74}$. Au moment où le premier télégraphe entre Francfort et Berlin est mis en service, l'histoire en tant que liée à l'écriture, de même que ses discours et documents, subissent la relève de l'âge des médias ${ }^{75}$. Avec l'introduction de la technique commença une nouvelle épochè que l'on n'atteint plus avec des analyses du discours, mais par une archéologie des médias. Les analyses du discours sont dépassées à l'âge des médias pour la bonne et simple raison que les histoires sont liées à l'écriture, tandis que se clôt le monopole de l'écrit ${ }^{76}$. Parce que les modes opératoires des médias techniques sont différents de l'écriture ou de la narration, ils échappent au regard fixé sur le texte. Parmi les conditions des médias techniques, les histoires du discours ne peuvent plus que tourner à vide parce que leurs objets contournent leur propre historicisation ${ }^{77}$.

On reconnaît la stratégie derrière cette argumentation de Kittler: en appliquant le geste d'historicisation de Foucault à Foucault, celui-ci est déclaré comme trop vieux pour la modernité médiatique et numérique ${ }^{78}$. De fait, le bibliothécaire n'a pas passé le plus clair de son temps dans des archives, mais dans des bibliothèques - raison pour laquelle le concept d'archive dans la pratique de la recherche foucaldienne correspond exactement à la bibliothèque ${ }^{79}$. Ce n'est pas par hasard que Foucault se sert de l'exemple de la machine à écrire et non de l'ordinateur pour illustrer sa théorie des énoncés ${ }^{80}$. Ordinateur et machine à écrire, c'est-à-dire le triangle indépassable de Grammophon-Film-Typewriter, vendent la mèche: l'opération fondamentale de l'archéologie des médias consiste à coupler l'histoire, les littératures et les discours aux développements techniques et médiatiques dont ils avaient été séparés au plus tard au début du $\mathrm{xx}^{\mathrm{e}}$ siècle par les sciences humaines. À la fin du siècle, les deux cultures sont enfin devenues une seule sous le signe du bien nommé cultural turn.

Pour autant, l'archéologie des médias n'a pas fait que restaurer la relation interrompue entre les sciences naturelles et leurs histoires des sciences oubliées. En plaçant la 
césure essentielle autour de 1850, elle place également ces dernières dans une constellation historique spécifique par rapport aux autres archéologies sauvages que sont l'archéologie de la modernité et l'archéologie du savoir. Tout d'abord, 1850 ne marque pas seulement la date à laquelle les médias techniques s'imposent - Kittler n'est jamais las de souligner que cette date est également celle à laquelle se terminent les analyses du discours de Foucault ${ }^{81}$. La correction méthodique de Kittler est donc consistante sur le plan historique, tant il est vrai que les analyses de l'accusé ne débordent pas sur les domaines que seule l'archéologie des médias est légitime à juger. Kittler travaille donc bien après et d'après Foucault : il ne travaille pas seulement au sens méthodique dans sa succession, soit d'après Foucault - il travaille surtout dans un sens historique après Foucault, puisque le domaine de recherche prolonge l'analyse du discours. Avec la césure de 1850, comme je l'ai souligné, pour Kittler c'est la fin du monopole de l'écriture et le début de l'empire de l'archéologie des médias.

D'une part l'argument méthodique est également couplé à un argument historique, d'autre part, la thèse historique d'une fin de l'analyse du discours fondée sur des documents porte déjà en soi l'argument de sa propre continuation méthodique en tant qu'archéologie des médias. L'argument historique est en cela lié à un argument méthodique qui met fin, avec l'émergence des médias techniques, au domaine d'action dans lequel opérait l'analyse foucaldienne du discours. Pour cette raison, l'archéologie des médias put se mettre en scène, comme cela a été dit, comme continuation historique et méthodique de l'archéologie du discours : elle la prolonge historiquement parce que, selon Kittler, «toutes» les études de Foucault se terminent en 1850; a contrario, si elles se terminent bien au milieu du XIX ${ }^{e}$ siècle, c'est que leur portée est épuisée méthodologiquement.

Kittler ne se contente donc pas d'un prolongement méthodique de Foucault. Ce dernier s'était refusé à historiciser son maître Canguilhem et avait évité de le classer dans une épistémè (ce que la position marquée par les sciences humaines de Canguilhem aurait certainement permis). Kittler en revanche conduit son historicisation de manière si radicale que même son témoin principal est historicisé, et ses concepts sont soumis à une reconstruction par l'archéologie des médias ${ }^{82}$. Comme auparavant Freud, Foucault n'est dorénavant plus un précurseur de l'archéologie des médias; lui aussi en est devenu l'objet, qui n'a pas survécu à la coupure entre l'époque des médias écrits et celle des médias techniques. Foucault fut certes «le dernier historien et le premier $\operatorname{archéologue~}^{83}$ ", comme l'écrit Kittler, qui fut, lui, le dernier archéologue de l'écrit et le premier des médias.

Kittler rend ainsi plausible son double prolongement de Foucault. La critique exercée à l'encontre de ce dernier est cependant plus problématique là où le lien se défait entre un argument méthodique et un argument historique. De fait, Kittler affûte son argument méthodique en construisant une confrontation universelle et méthodique entre des analyses du texte ou des techniques ${ }^{84}$. Si l'on objecte de manière générale à Foucault de ne mettre l'accent que sur le discours, de n'être centré que sur la bibliothèque, et si l'on déplore l'absence complète des médias, on oublie que l'analyse du discours avait son domaine de compétence avant 1850 . Le reproche contre le philosophe Foucault est donc de n'être pas encore parvenu à l'analyse médiatique du savoir, que l'on ne pratique toutefois que sur la base qu'il a mise au jour. Ici, on ne peut que recommander au nain sur les épaules du géant de regarder vers le bas, vers les 
conditions théoriques et historiques qui ont formé son regard, ce que firent les archéologies sauvages.

\section{BIBLIOGRAPHIE}

Bolz Norbert, Philosophie nach ihrem Ende, Munich, Boer, 1992.

Dosse François, Geschichte des Strukturalismus, 2 vol., Francfort-sur-le-Main, Fischer-TaschenbuchVerlag, 1999.

Dotzler Bernhard, Diskurs und Medium. Zur Archäologie der Computerkultur, Munich, Wilhelm Fink, 2006.

Ebeling Knut, «Das technische Apriori », in Kulturgeschichte als Mediengeschichte (oder vice versa ?), Lorenz Engell, Bernhard Siegert, Joseph Vogl (eds), Weimar, Universitätsverlag, 2006.

---, Wilde Archäologien, Wilde Archäologien 1. Theorien der materiellen Kultur von Kant bis Kittler, Berlin, Kadmos, 2012.

Ernst Wolfgang, Das Rumoren der Archive. Ordnung aus Unordnung, Berlin, Merve, 2002.

-- , Im Namen von Geschichte: Sammeln - Speichern - Er/zählen. Infrastrukturelle Konfigurationen des deutschen Gedächtnisses, Munich, Wilhelm Fink, 2003.

- - , « Das Gesetz des Sagbaren. Foucault und die Medien », in Foucault und die Künste, Peter Gente (ed.), Francfort-sur-le-Main, Suhrkamp, 2004.

-—-, « Diskurs und Medien. Michel Foucault, medienarcheologisch gelesen », in Quadratur Kulturbuch Nr. 5/Medien, Marcus Kleiner et Holger Ostwald (eds.), Aschaffenburg/Duisburg, 2004.

Flusser Vilém, « Räume », in Raumtheorie. Grundlagentexte aus Philosophie und Kulturwissenschaften, Jörg Dünne et Stephan Günzel (eds), Francfort-sur-le-Main, Suhrkamp, 2006.

Foucault Michel, «Préface à l'édition anglaise », in The Order of Things, Londres, Tavistock, 1970.

-_-, « Le philosophe masqué », in Dits et Écrits. 1954-1988, vol. IV (1980-1988), Paris, Gallimard, coll. « NRF », 1994, p. 104-110.

-_-, « L'imagination au XIX siècle », in Dits et Écrits. 1954-1988, vol. IV (1980-1988), Paris, Gallimard, coll. « NRF », 1994, p. 111-115.

Groys Boris, Unter Verdacht. Eine Phänomenologie der Medien, Munich, Hanser, 2000.

Hartmann Frank, « Friedrich Kittler », in Information Philosophie, nº 4, 1997.

Kittler Friedrich, «Ein Verwaiser », in Anschlüsse. Versuche nach Michel Foucault, Gesa Dane et Wolfgang Eßbach (eds), Tubingen, Edition Diskord, 1985.

--_, Grammophon, Film, Typewriter, Berlin, Brinkman \& Bose, 1986.

-_-, Dichter, Mutter, Kind, Deutsche Literatur im Familiensystem 1760, Munich, Wilhelm Fink, 1991. 
---, « Synergie von Mensch und Maschine. Ein Gespräch mit Florian Rötzer », in Kunst machen? Gespräche über die Produktion von Bildern, Florian Rötzer et Stefan Rogenhofer (eds), Leipzig, Reclam, 1993.

-_-, Draculas Vermächtnis. Technische Schriften, Leipzig, Reclam, 1993.

-—-, « Die Evolution hinter unserem Rücken », in Kultur und Technik im 21. Jahrhundert, Gerhard Kaiser (ed.), Francfort-sur-le-Main, Suhrkamp, 1993.

-_-, « Wenn die Freiheit wirklich existiert, dann soll sie doch ausbrechen », in Am Ende vorbei, Rudolf Maresch, Vienne, Turia und Kant, 1994.

--_, Aufschreibesysteme 1800/1900, Munich, Wilhelm Fink, 1995.

-—-, «Zum Geleit », in Der Foucault-Reader: Diskurs und Medien, Jan Engelman (ed.), Stuttgart, Deutsche Verlags-Anstalt, 1999.

-_-, Short Cuts, Francfort-sur-le-Main, Zweitausendeins, 2002.

Kittler Friedrich et Horst Turk, "Vorwort der Herausgeber und Einleitung », in Urszenen.

Literaturwissenschaft als Diskursanalyse und Diskurskritik, Francfort-sur-le-Main, Suhrkamp, 1977.

Kittler Friedrich et al., « Das Beste, was du wissen kannst », in Diskursanalysen 2. Institution Universität, Friedrich Kitter, Manfred Schneider et Samuel Weber (eds), Opladen, Westdeutscher Verlag, 1990.

Krämer Sybille, « Friedrich Kittler - Kulturtechniken der Zeitachsenmanipulation », in Medientheorie. Eine philosophische Einführung, Alice Lagaay et David Lauer (eds), Francfort-sur-leMain, Campus, 2004.

Krauss Rosalind, The Optical Unconscious [1994], trad. par Michèle Veubret, Paris, Au même titre, 2002.

Ronell Avital, Das Telefonbuch. Technik, Schizophrenie, Elektrische Rede, Berlin, Brinkmann \& Bose, 2001.

Schneider Ulrich Johannes, Michel Foucault, Darmstadt, Wissenschaftliche Buchgesellschaft, 2004.

Winthrop-Young Geoffrey, Friedrich Kittler zur Einführung, Hambourg, Junius, 2005.

\section{NOTES}

1. L'exposition Archéologie de l'image numérique. Les débuts de l'art informatique à la Kunsthalle de Brême (Brême, 2006/2007) en fournit un exemple.

2. Winthrop-Young souligne, dans la première présentation complète de l'entreprise kittlérienne, le caractère controversé de celle-ci. Cf. Winthrop-Young Geoffrey, Friedrich Kittler zur Einführung, Hambourg, Junius, 2005.

3. Cf. Kittler Friedrich A., "Exorciser l'homme des sciences humaines: programmes du poststructuralisme », Appareil [En ligne], | 2017, mis en ligne le 13 décembre 2017, consulté le 13 décembre 2017. URL : http://journals.openedition.org/appareil/2432

4. Winthrop-Young Geoffrey, Friedrich Kittler zur Einführung, , p. 62.

5. Kittler Friedrich, Dichter, Mutter, Kind, Munich, Wilhelm Fink, 1991, p. 14.

6. «Le rapport entre l'histoire de la culture et la science historique des médias apparait comme une guerre de succession larvée pour le trône vacant de l'a priori.» Cf. Kulturgeschichte als Mediengeschichte (oder vice versa?), Lorenz Engell, Bernhard Siegert, Joseph Vogl (eds), Weimar, Universitätsverlag, 2006, p. 7. 


\section{Ibid., p. 8.}

8. Ebeling Knut, « Das technische Apriori », in Kulturgeschichte als Mediengeschichte (oder vice versa?), Lorenz Engell, Bernhard Siegert, Joseph Vogl (eds), Weimar, Universitätsverlag, 2006.

9. L'auteur entend par "archéologie sauvage " une pensée qui, de Kant à Kittler, se serait opposée à une réflexion de type historique pour en questionner les conditions de possibilité ou l'a priori. Là où l'histoire impose le réalisme du "ça s'est passé ainsi » (Ebeling Knut, Wilde Archäologien, Wilde Archäologien 1. Theorien der materiellen Kultur von Kant bis Kittler, Berlin, Kadmos, 2012, p. 15.), l'archéologie consiste en une enquête sur les traces matérielles; ainsi, elle ne représente pas tant le passé qu'elle le " matérialise » (ibid.). Partant de là, Ebeling propose une réflexion sur l'archéologie de la métaphysique chez Kant, sur l'archéologie de la psyché par Freud, sur l'archéologie de la modernité de Benjamin, l'archéologie du savoir de Foucault et enfin l'archéologie des médias avec Kittler. [N.d.T.]

10. Ernst Wolfgang, "Das Gesetz des Sagbaren. Foucault und die Medien ", in Foucault und die Künste, Peter Gente (ed.), Francfort-sur-le-Main, Suhrkamp, 2004, p. 243.

11. Winthrop-Young G., Friedrich Kittler zur Einführung, op. cit., p. 27.

12. Le titre original signifie littéralement: «Changements structurels de l'espace public». [N.d.T.]

13. Kittler F., Draculas Vermächtnis. Technische Schriften, Leipzig, Reclam, 1993, p. 150.

14. Winthrop-Young G., Friedrich Kittler zur Einführung, op. cit., p. 97. Concernant le phénomène de conversion de chercheurs en lettres à la théorie des médias, on se reportera aux pages 80 et suivantes du même ouvrage.

15. «Plus la technique se distingue de l'homme et le laisse derrière elle, plus elle agit de manière déterminante sur lui ». Ibid., p. 78. [Nous traduisons les citations.]

16. Ibid., p. 231. Plusieurs techniques mathématiques consistent à réduire «des infinités incalculables" en «quantités finies calculables» (ibid., p. 194) pour faire de ces éléments incalculables des choses avec lesquelles on peut calculer - raison pour laquelle le concept technique de code comme "quantité dénombrable d'éléments» est au centre de ces calculs. L'expression « quantité dénombrable d'éléments » est tirée de Kittler F., Short Cuts, Francfort-surle-Main, Zweitausendeins, 2002, p. 92.

17. Cf. Dotzler Bernhard, Diskurs und Medium. Zur Archäologie der Computerkultur, Munich, Wilhelm Fink, 2006.

18. À la suite de Kittler, l'élaboration par Groys d'un « espace sub-médiatique » et par W. Ernst d'une "an-aisthesis» visent ce fond enfoui des médias. Cf. Groys B., Unter Verdacht. Eine Phänomenologie der Medien, Munich, Hanser, 2000, p. 19, et Ernst W., « Das Gesetz des Sagbaren. Foucault und die Medien », op. cit., p. 241.

19. «Derrière les surfaces médiatiques, il n'y a pas de secrets mais des algorithmes et des composantes de machines - il ne s'agit que de savoir les lire» (Ernst W., «Das Gesetz des Sagbaren. Foucault und die Medien», op. cit., p. 241.) Concernant le geste démystifiant et désenchanté de l'archéologie des médias, voir Winthrop-Young G., Friedrich Kittler zur Einführung, op. cit., p. 91.

20. Winthrop-Young G., Friedrich Kittler zur Einführung, op. cit., p. 87. Concernant les corrections apportées par Foucault à Freud, voir le même p. 91.

21. Concernant le triangle Foucault, Lacan et Kittler, voir F. Kittler, Grammophon, Film, Typewriter, Berlin, Brinkmann \& Bose, 1986, p. 27, et G. Winthrop-Young, Friedrich Kittler zur Einführung, op. cit., p. 41.

22. Cf. Kittler F., Dichter, Mutter, Kind, op. cit. 
23. Cf. Kittler F., «Das Beste, was du wissen kannst ", in Diskursanalysen 2. Institution Universität, Friedrich Kitter, Manfred Schneider et Samuel Weber (eds), Opladen, Westdeutscher Verlag, 1990.

24. Benjamin Walter, Gesammelte Schriften, Rolf Tiedemann et Hermann Schweppenhäuser (eds), Francfort-sur-le-Main, Suhrkamp, 1977, vol. II, p. 371. Cf. Rosalind Krauss, L'Inconscient optique [1994], trad. par Michèle Veubret, Paris, Au même titre, 2002.

25. Foucault Michel, "Préface à l'édition anglaise ", in The Order of Things, Londres, Tavistock, 1970, p. 10.

26. Cf. Kittler F., Dichter, Mutter,Kind, op. cit.

27. Le concept de «système de prise de notes » ou, en allemand, de «Aufschreibesysteme " a été élaboré par Kittler dans sa thèse d'habilitation parue sous le titre éponyme: Aufschreibesysteme 1800/1900. L'expression elle-même est empruntée aux Mémoires d'un névropathe de Daniel Paul Schreber et permet à Kittler de décrire différentes configurations historiques de la connexion entre médias (institutions ou techniques) et corps humains. [N.d.T.]

28. Kittler Friedrich et Horst Turk, "Vorwort der Herausgeber und Einleitung ", in Urszenen. Literaturwissenschaft als Diskursanalyse und Diskurskritik, F. Kittler et H. Turk (eds), Francfort-surle-Main, Suhrkamp, 1977.

29. Kittler F., Draculas Vermächtnis. Technische Schriften, op. cit., p. 63. Sur le rapport de Kittler à Freud, voir G. Winthrop-Young, Friedrich Kittler zur Einführung, op. cit., p. 89.

30. Kittler F., Draculas Vermächtnis. Technische Schriften, op. cit., p. 63.

31. «Si [l'analyse du discours] établit des références, elle ne vise pas pour autant des états de choses mais rapporte des discours à des discours" (Kittler F. et H. Turk, "Vorwort der Herausgeber und Einleitung ", op. cit., p. 40.)

32. Kittler F., Draculas Vermächtnis. Technische Schriften, op. cit., p. 8.

33. Au regard du caractère lisse des codages immatériels, on pourrait soupçonner un retour au structuralisme qui avait défini le code dans les termes des techniques de la communication : «La définition du code est d'être traduisible en un autre code : cela définit les propriétés de ce qu'on appelle "structure" ", Dosse François, Geschichte des Strukturalismus, 2 vol., Francfort-sur-le-Main, Fischer-Taschenbuch-Verlag, 1999, p. 58.

34. Winthrop-Young G., Friedrich Kittler zur Einführung, op. cit., p. 138.

35. Cf. Flusser Vilém, "Räume", in Raumtheorie. Grundlagentexte aus Philosophie und Kulturwissenschaften, Jörg Dünne et Stephan Günzel (eds), Francfort-sur-le-Main, Suhrkamp, 2006, p. 274-288.

36. Kittler F., Draculas Vermächtnis. Technische Schriften, op. cit., p. 8.

37. Ibid., p. 9.

38. «L'archéologie des médias cherche [...] à saisir la loi médiatique qui guide l'ordre des choses dans leur production même » (Ernst W., "Das Gesetz des Sagbaren. Foucault und die Medien », op. cit., p. 169.)

39. Kittler F., Short Cuts, op. cit., p. 36.

40. Kittler F., « Die Evolution hinter unserem Rücken », in Kultur und Technik im 21. Jahrhundert, Gerhard Kaiser (ed.), Francfort-sur-le-Main, Suhrkamp, 1993.

41. Cf. Ronell Avital, Das Telefonbuch. Technik, Schizophrenie, Elektrische Rede, Berlin, Brinkmann \& Bose, 2001.

42. Winthrop-Young G., Friedrich Kittler zur Einführung, op. cit., p. 77.

43. Kittler F., Short Cuts, op. cit., p. 91.

44. Kittler F., «Wenn die Freiheit wirklich existiert, dann soll sie doch ausbrechen », in Am Ende vorbei, Rudolf Maresch (ed.), Vienne, Turia und Kant, 1994, p. 114.

45. Winthrop-Young G., Friedrich Kittler zur Einführung, op. cit., p. 77. 
46. Ibid., p. 26.

47. Ibid., p. 77 et Krämer Sybille, «Friedrich Kittler - Kulturtechniken der Zeitachsenmanipulation », in Medientheorie. Eine philosophische Einführung, Alice Lagaay et David Lauer (eds), Francfort-sur-le-Main, Campus, 2004, p. 201.

48. Cf. Ernst W., "Diskurs und Medien. Michel Foucault, medienarcheologisch gelesen ", in Quadratur Kulturbuch Nr. 5/Medien, Marcus Kleiner et Holger Ostwald (eds), Aschaffenburg/ Duisburg, 2004, p. 170.

49. Foucault M., « Préface à l'édition anglaise » de Les Mots et les choses, op. cit., p. 10.

50. Cf. note 27. [N.d.T.]

51. Kittler F., Aufschreibesysteme 1800/1900, Munich, Wilhelm Fink, 1995, p. 9.

52. Winthrop-Young G., Friedrich Kittler zur Einführung, op. cit., p. 86.

53. Cf. Kittler F., "Ein Verwaiser", in Anschlüsse. Versuche nach Michel Foucault, Gesa Dane et Wolfgang Eßbach (eds), Tubingen, Edition Diskord, 1985, et « Zum Geleit », in Der Foucault-Reader: Diskurs und Medien, Jan Engelman (ed.), Stuttgart, Deutsche Verlags-Anstalt, 1999.

54. Friedrich K., "Zum Geleit », op. cit., p. 9.

55. Foucault M., «Le philosophe masqué », in Dits et Écrits. 1954-1988, vol. IV (1980-1988), Paris, Gallimard, coll. « NRF », 1994, p. 104-110.

56. Foucault M., « L'Imagination au XIX ${ }^{\mathrm{e}}$ siècle », in Dits et Écrits, op. cit., p. 111-115.

57. Kittler F., « Ein Verwaiser », op. cit., p. 141.

58. Selon ses propres dires, Kittler n'avait pas osé adresser la parole à Foucault à Bayreuth en 1980 (cf. « Ein Verwaiser », op. cit., p. 141).

59. Kittler rend compte de cette avidité en décrivant la manière dont il attendait les livres de Foucault, comme on attend « les nouveaux disques de rock » (Kittler F., « Ein Verwaiser », op. cit., p. 141.)

60. Kittler F., «Ein Verwaiser », op. cit., p. 8 ; Ernst W., «Diskurs und Medien. Michel Foucault, medienarcheologisch gelesen », op. cit., p. 168 ; Krämer S., « Friedrich Kittler - Kulturtechniken der Zeitachsenmanipulation ", op. cit., p. 207.

61. Kittler F., Draculas Vermächtnis. Technische Schriften, op. cit., p. 222.

62. Ibid., p. 10.

63. Ibid., p. 169.

64. Kittler F., Short Cuts, op. cit., p. 39.

65. Ernst W., «Diskurs und Medien. Michel Foucault, medienarcheologisch gelesen », op. cit., p. 168 ; Winthrop-Young G., Friedrich Kittler zur Einführung, op. cit., p. 108. Winthrop-Young ne se contente pas de souligner le handicap qu'est la tache aveugle, mais également ce que cette dernière rend possible : «La science kittlérienne des médias apparaît comme une continuation conséquente des travaux de Foucault, en cela qu'elle rend visible sa tache aveugle sous la forme d'un a priori médiatique ».

66. Winthrop-Young G., Friedrich Kittler zur Einführung, op. cit., p. 83.

67. Kittler F., « Ein Verwaiser », op. cit., p. 8.

68. Norbert Bolz également souligne la «séquence paradigmatique évidente" menant du structuralisme à la théorie des médias en passant par l'analyse du discours (Bolz N., Philosophie nach ihrem Ende, Munich, Boer, 1992.)

69. Kittler F., Draculas Vermächtnis. Technische Schriften, op. cit., p. 10.

70. Winthrop-Young G., Friedrich Kittler zur Einführung, op. cit., p. 109.

71. Ernst W., "Diskurs und Medien. Michel Foucault, medienarcheologisch gelesen », op. cit., p. 168.

72. Cf. Kittler F., "Ein Verwaiser", op. cit.; Ernst W., Das Rumoren der Archive. Ordnung aus Unordnung, Berlin, Merve, 2002; Im Namen von Geschichte: Sammeln - Speichern - Er/zählen. Infrastrukturelle Konfigurationen des deutschen Gedächtnisses, Munich, Wilhelm Fink, 2003 ; « Diskurs und Medien. Michel Foucault, medienarcheologisch gelesen », op. cit. 
73. Kittler F. et Horst Turk, « Vorwort der Herausgeber und Einleitung », op. cit., p. 19.

74. Kittler F., Grammophon - Film - Typewriter, op. cit., p. 12.

75. Ibid. p. 33.

76. Kittler F., Aufschreibesysteme 1800/1900, op. cit., p.519; F. Kittler, Grammophon - Film Typewriter, op. cit., p. 13. «La nécessité d'une théorie des médias n'apparaît que dans l'envers du livre (Hartmann Frank, « Friedrich Kittler », in Information Philosophie, $\mathrm{n}^{\circ}$ 4, 1997, p. 42.) Ainsi « la portée de l'analyse foucaldienne du discours se trouve limitée dans le temps et dans ses objets ; elle se limite à l'époque où le médium dominant était l'écrit imprimé, et même pour cette période-là, elle doit être complétée par l'analyse des médias » (Winthrop-Young G., Friedrich Kittler zur Einführung, op. cit., p. 86.)

77. «Là où la réalité technique suspend la différenciation des médias, la conceptualité également perd tout assise » (Winthrop-Young G., Friedrich Kittler zur Einführung, op. cit., p. 140.)

78. Cf. Krämer S., «Friedrich Kittler - Kulturtechniken der Zeitachsenmanipulation », op. cit., p. 207. On trouve chez Schneider une proposition alternative à cette historicisation médiahistorique du travail de Foucault, notamment lorsqu'il souligne la manière dont Foucault «crée de nouveaux liens [...] en groupant les livres de manière nouvelle " (Schneider U. J., Michel Foucault, Darmstadt, Wissenschaftliche Buchgesellschaft, 2004, p. 97.)

79. Kittler, dans ses Aufschreibesysteme 1800/1900, (op. cit., p. 519.) se distingue ici de Ernst (cf. Das Rumoren der Archive. Ordnung aus Unordnung, op. cit.) qui tente de restituer la matérialité de l'archive. Concernant la question de la bibliothèque, voir Schneider Ulrich Johannes, Michel Foucault, op. cit, qui entreprend de faire un portrait instantané monumental de la bibliothèque classique. Cf. également Winthrop-Young G., Friedrich Kittler zur Einführung, op. cit., p. 84.

80. Kittler F., « Ein Verwaiser », op. cit., p. 145 ; Ernst W., « Das Gesetz des Sagbaren. Foucault und die Medien », op. cit., p. 250.

81. «[Les] analyses historiques [de Foucault] s'arrêtent toutes pratiquement au moment où d'autres médias et d'autres techniques de transmission entament la chasse-gardée des livres. Pour les archives sonores et les tours de bobines de films, l'analyse du discours n'est plus compétente» (Kittler F., Grammophon - Film - Typewriter, op. cit., p. 13.) «La seule analyse des discours n'épuise pas les formes du pouvoir et du savoir ». (Kittler F., Aufschreibesysteme 1800/1900, op. cit., p. 519.) Cette thèse de Kittler trouva une approbation certaine: «Une archéologie des formes contemporaines du savoir [ne peut] plus être conduite comme analyse du discours, mais doit être étendue à une analyse des médias techniques. [...] Les enquêtes média-historiques de Kittler commencent là où les enquêtes de Foucault cessent ». (Krämer F., «Friedrich Kittler Kulturtechniken der Zeitachsenmanipulation», op. cit., p. 207.) «L'archéologie du savoir de Kittler embraye là où les analyses du discours de Foucault s'arrêtent » (Ernst W., "Diskurs und Medien. Michel Foucault, medienarcheologisch gelesen », op. cit., p. 170.)

82. «Kittler applique l'archéologie du savoir à Foucault lui-même » (Ernst W., «Diskurs und Medien. Michel Foucault, medienarcheologisch gelesen », op. cit., p. 170.)

83. Kittler F., Grammophon - Film - Typewriter, op. cit., p. 13.

84. Ernst parle d'un «combat entre une rhétorique linguistique et une technique commutée, entre des bibliothèques fondées sur le livre ou au contraire sur les données électroniques. (Ernst W., « Diskurs und Medien. Michel Foucault, medienarcheologisch gelesen », op. cit., p. 168-175, et «Das Gesetz des Sagbaren. Foucault und die Medien », op. cit., p. 225.) 


\section{RÉSUMÉS}

$\mathrm{Au} \mathrm{Xx}^{\mathrm{e}}$ siècle, les avant-gardes intellectuelles furent souvent archéologiques. Archéologie du psychisme chez Freud, du discours chez Foucault ou enfin des médias chez Kittler. Adoptant le point de vue de ce dernier, l'article interroge les continuités et les ruptures entre ces penseurs. Des projets freudien et foucaldien, Kittler fait tout d'abord surgir le refoulé technique avant de les soumettre à une critique historique radicale - Foucault se voyant, en dernier lieu, confier le rôle d'archiviste de l'âge classique. Ayant en effet échoué à poser la question des médias techniques, ce dernier ne pouvait dépasser dans ses analyses le milieu du XIX $x^{e}$ siècle - ce à quoi l'on répondra à Kittler que puisque Foucault n'a pas dépassé le milieu du XIX siècle, il n'avait pas besoin d'aborder les médias techniques. La question dépasse cependant la simple polémique; elle engage la définition de l'a priori qui, de «transcendantal » chez Kant était devenu « historique » chez Foucault jusqu'à devenir « média-technique » chez Kittler - au risque de retrouver un statut quasi-transcendental.

\section{INDEX}

Mots-clés : a priori média-technique, psychanalyse, analyse du discours, archéologie des médias

\section{AUTEURS}

\section{KNUT EBELING}

Knut Ebeling est professeur de Théorie des médias et d'esthétique à la Kunsthochschule Weißensee de Berlin. Il a été chargé de cours à la Stanford University de Berlin et a enseigné à l'École supérieure des Beaux-Arts d'Alger. Depuis sa thèse de doctorat écrite sous la direction de Friedrich Kittler, Die Falle. Zwei Lektüren zu Georges Batailles « Madame Edwarda » (Passagen-Verlag, 2000), il s'est intéressé aux théories de l'archive (Archivologie, Kadmos, 2009 ; Das Archiv brennt, Kadmos, 2007, en collaboration avec Georges Didi-Hubermann) et à l'archéologie des médias dans une perspective matérialiste (Wilde Archäologien 1. Theorien der materiellen Kultur von Kant bis Kittler, Kadmos 2012, et Wilde Archäologien 2. Begriffe der Materialität der Zeit - von Archiv bis Zerstörung, 2016). 